research article

\title{
Quality of life in patients after combined modality treatment of rectal cancer: Report of a prospective phase II study
}

\author{
Vaneja Velenik, Irena Oblak, Franc Anderluh \\ Department of Radiotherapy, Institute of Oncology Ljubljana, Slovenia
}

\begin{abstract}
Background. The literature reports are unclear whether a permanent stoma reduces the quality of life (QOL) of patients with locally advanced rectal cancer (T3-4 and/or N+). Our aim was to compare the QLQ of patients with abdominoperineal resection and with restorative surgery, treated with preoperative radiochemotherapy in a prospective phase II clinical trial.

Methods. Fifty-seven patients were irradiated to $45 \mathrm{~Gy}$ in 25 fractions over 5 weeks to the pelvis concomitantly with oral capecitabine $825 \mathrm{mg} / \mathrm{m}^{2}$, twice a day, including weekends. Surgery was scheduled 4-6 weeks after the completion of the chemoradiotherapy. Four courses of chemotherapy were planned postoperatively. Patients still alive and without recurrence of the disease, with a minimum follow up of 2 years, were surveyed with two self-rating questionnaires developed by the European Organisation for Research and Treatment of Cancer (EORTC): one was cancer specific (EORTC QLQ-C30) and one was site specific (EORTC QLQ-C38).
\end{abstract}

Results. QLQ was assessed in 28 of 32 patients eligible (87.5\%). The median time from surgery to filling in the questionnaires was 35 months. For all scales of EORTC QLQ-C30 and EORTC QLQ-C38, no significant differences in median scores were observed between the two groups of patients.

Conclusions. QOL did not differ in patients with abdominoperineal resection from patients with sphinctersparing surgery.

Key words: preoperative radiochemotherapy; rectal cancer; quality of life

\section{Introduction}

The preoperative chemoirradiation has become a standard part of treatment protocols in stage II and III rectal cancer. Compared

Received 13 October 2008

Accepted 5 November 2008

Correspondence to: Vaneja Velenik, PhD, MD, Department of Radiotherapy, Institute of Oncology Ljubljana, Zaloška c. 2, 1000 Ljubljana, Slovenia. Phone: +38615879 661; Fax: +386 15879 304; E-mail: vvelenik@onko-i.si to postoperative chemoradiotherapy, the advantage of preoperative application of chemotherapeutics and irradiation includes improved compliance, reduced toxicity and down staging of the tumour in a substantial number of patients. The latter can potentially increase the feasibility of sphincter-saving resection in low-sited tumours. ${ }^{1}$ The impairment of anorectal, voiding and sexual function is a frequent adverse effect of the multimodality treatment. Thus, the addition of radiotherapy (RT) to surgery 
improves the oncologic outcome but, potentially, adds morbidity to that associated with surgery. ${ }^{2}$ A poor functional outcome after the restorative surgical technique may effect on the patient's quality of life (QOL). The construction of permanent colostomy following an abdominoperineal resection (APR) may be associated with one or more physical and psyhosociological problems as well. In the past, prospective studies with rectal cancer patients have focused on the tumour response, local control, survival and treatment related toxicity as primary end-points. Although these parameters remain central in the evaluation process, there is an increasing recognition of the need to assess more systematically the impact of cancer and its treatment on the functional, psychological and social health of the individual. As MRI has become incorporated in the diagnostic procedure of rectal disease, ${ }^{3}$ the measurement of the functional outcome and QOL in rectal cancer patients, treated with preoperative radiochemotherapy and surgery has also become incorporated in clinical trials over the past decade. ${ }^{4}$

The aim of the present study was to assess QOL outcomes in patients treated with restorative procedures, compared with those in patients after APR by using a recommended and proven method.

\section{Patients and methods}

\section{Patients}

Between June 2004 and January 2005, fiftyseven patients with locally advanced resectable rectal cancer were treated with preoperative radiotherapy and concomitant capecitabine. Thirty-two patients, who were alive and without evidence of disease progression at a minimum follow-up of 2 years, were asked to participate in QOL
Table 1. Characteristics of patients who answered the questionnaires

\begin{tabular}{lr}
\hline Characteristics & Number (\%) \\
\hline $\begin{array}{l}\text { Number of responders/eligible } \\
\text { patients }\end{array}$ & $28 / 32(87.5)$ \\
Median age (range) years & $67(37-81)$ \\
Gender & \\
$\quad$ Male & $20(71.4)$ \\
Female & $8(28.6)$ \\
WHO performance status & \\
$\quad$ Stage 0 & $27(96.4)$ \\
$\quad$ Stage I & $1(3.6)$ \\
Tumour distance from the anal & $6.5(1-12)$ \\
verge (cm) & \\
Clinical TNM stage & $14(50.0)$ \\
Stage II & $14(50.0)$ \\
Stage III & \\
Permanent stoma & $9(32.1)$ \\
Yes & $19(67.9)$ \\
$\quad$ No & \\
Postoperative chemotherapy & \\
Yes & $27(96.4 \%)$ \\
No & $1(3.6 \%)$ \\
Median time (range) from surgery \\
to answering (ra
\end{tabular}

study. Twenty-eight participated after having given informed consent. The characteristics of patients who answered the questionnaires are listed in Table 1.

\section{Treatment}

The details of both the patients and the treatment have been reported previously. ${ }^{5}$ Briefly, the prospective phase II trial has been approved by the Republic Ethic Committee. The entry criteria included: histologically verified adenocarcinoma of the rectum, clinical stage II or III (IUCC TNM classification 2002); no prior radiotherapy and/or chemotherapy; World Health Organisation (WHO) performance 
status <2; age at diagnosis of 18 or older; adequate bone marrow, liver, renal and cardiac function (no history of ischemic heart disease), and written informed consent.

Radiotherapy was delivered using 15 MV photon beams and four-field box technique, once per day, 5 days weekly. The small pelvis received 45 Gy in 25 fractions over 5 weeks. Three-dimensional CT-based treatment planning was performed. The clinical target volume (CTV) was defined to cover the small pelvis from the L5-S1 interspace to $5 \mathrm{~cm}$ below the primary tumour. The lateral borders were $5 \mathrm{~mm}$ outside the true bony pelvis. The posterior margin covered the sacrum, and the anterior margin encompassed the posterior one-third to one-half of the bladder and/or vagina. An additional $1 \mathrm{~cm}$ in all directions was added to the CTV to obtain the planning target volume (PTV). The dose was prescribed to cover the PTV with a $95 \%$ reference isodose $(95 \%$ of the ICRU point dose). Patients were treated in the prone position. They were instructed to have a full bladder during irradiation, and no devices were used to displace the small bowel out of the irradiated volume. A multileaf collimator was used for shaping the fields and for the protection of normal tissues.

Chemotherapy was administered concomitantly with radiotherapy and consisted of capecitabine administered orally at a daily dose of $1650 \mathrm{mg} / \mathrm{m}^{2}$, divided into two equal doses given 12 hours apart. One of the doses was taken 2 hours prior to irradiation. The chemotherapy started on the first day of radiotherapy and finished on the last day of radiotherapy (including weekends).

According to the protocol, surgery was planned for 4-6 weeks after the completion of the chemoradiotherapy. Although TME was the preferred surgical technique, it was not mandatory. Abdominoperineal resection (APR) was carried out in 17 (30.9\%) patients, anterior resection (AR) in $4(7.3 \%)$ patients, low anterior resection (LAR) in 32 $(58.2 \%)$ patients, exenteration of the small pelvis in $1(1.8 \%)$ patient and Hartmann's resection in $1(1.8 \%)$ patient. As determined by the histopathological examination of surgical specimens, the resection was radical (R0) in 54 (98.2\%) patients. A temporary colostomy was required in $32(88.8 \%)$ patients.

Four courses of chemotherapy were planned postoperatively. It was administered in 44/55 (80\%) of patients. Eighteen $(40.9 \%)$ patients received adjuvant 5Fluorouracil/Leukovorin and 26 (59.1\%) patients received capecitabine.

\section{QLQ assessment}

QLQ was assessed using two validated questionnaires developed by European Organisation for Research and Treatment of Cancer (EORTC). One questionnaire assessed the cancer specific QOL (the third version of the Quality of life Questionnaire Core 30 items, i.e. QLQ-C30) ${ }^{6}$ and the other site - specific (colorectal) QOL (Quality of life Questionnaire Core 38 items, i.e. QLQ-C38).7

The QLQ-C30 is a 30-items questionnaire. It includes a total of nine multi-item scales: five functional scales (physical, role, cognitive, emotional, and social); three symptom scales (fatigue, pain, and nausea and vomiting); and a global health and quality-of-life scale. Separate six single items are included to measure gastrointestinal symptoms (diarrhoea and constipation), dyspnoea, appetite loss, sleeping disturbances and economic consequences of the disease.

The QLQ-C38 questionnaire comprises 38 questions, of which 19 are completed by all patients and the remaining by a subset of the patients (men or women; patients with or without stoma). It incorporates two functional scales (body image and sexuality) and seven symptom scales (micturition 
problems induced by irradiation, chemotherapy side effects, gastrointestinal general symptoms, defecation problems, stoma - related problems, and sexual dysfunction in men and women). The remaining single items assess future perspectives and weight loss.

Both questionnaires contain questions related to the previous week. Four response categories, from 1 (not at all) to 4 (very much), are possible.

\section{Statistical analysis}

The scoring was performed according to the EORTC QLQ-C30 scoring manual. ${ }^{8}$ The principle of scoring was to estimate the average of the items that contributed to the scale; this was the raw score. A linear transformation was used to standardize the raw score, so that all scores ranged from 0 to 100 . The higher scale score for the functional scale or the global health status/QOL represents a higher level of functioning, or higher QOL; whereas the higher level of symptoms/problems for the symptom/ item scales represents a higher level of symptomatology, or dysfunction. Missing values were calculated such that if at least one-half the items from the scale had been completed, it was assumed that the missing items would have had values equal to the average of the items present.

Demographic and clinical data were calculated using descriptive statistics. Results of QOL information were expressed as means and medians. The nonparametric Mann-Whitney U-test was used to compare median scores of QOL scales between the two treatment groups of patients. A 5\% level of statistical significance was used for variables $(P<0.05)$. Data were analyzed using SPSS for Windows (version 13.0; SPSS, Chicago, IL, USA).

We hypothesized that at least some scores of various scales would vary between subgroups of patients in favour of patients with restorative type of surgery.

\section{Results}

\section{Oncological outcome}

Before the therapy, an abdominoperineal resection was planned in 24 out of 55 patients who had definitive surgery. After the completion of chemoradiotherapy, the sphincter-conserving surgery was successfully performed in 7 of these 24 patients. Among 31 patients in whom the sphincterconserving surgery was planned before having had any therapy, this was not possible in two patients, which resulted in an ultimate sphincter preservation rate of $65.5 \%(36 / 55)$.

A local relapse has occurred in 1 (1.8\%) patient and a dissemination in 13 (24.1\%) out of 54 patients with a median time to progression of 23 months (range 3-23 months). Second malignancies have occurred in 2 patients. The median 2-year overall survival, disease-free survival and disease-specific survival rates were $84.2 \%, 72.5 \%$ and $92.4 \%$, respectively, and local control was $98.2 \%$.

\section{QOL evaluation}

Of 32 eligible patients from the prospective phase II trial, 28 (87.5\%) completed the EORTC QLQ-C30 and QLQ-C38 questionnaires: 19 patients with sphincter conserving surgery and 9 patients with APR. Three patients refused to participate in the study and one was judged ineligible because of serious comorbidities. Surveys were completed a median of 35 (26-39) months after the surgery.

The general results of QLQ-C30 for all patients with or without stoma are given in Table 2. The global quality of life scores, representing the overall health and quality 
Table 2. EORTC QLQ-C30 mean and median functional scale and single-item scores according to the type of surgery

\begin{tabular}{|c|c|c|c|c|c|}
\hline \multirow[b]{2}{*}{ Item } & \multicolumn{2}{|c|}{$\begin{array}{c}\text { APR } \\
\text { (8 patients) }\end{array}$} & \multicolumn{2}{|c|}{$\begin{array}{l}\text { Restorative surgery } \\
\text { (20 patients) }\end{array}$} & \multirow[b]{2}{*}{$P$} \\
\hline & Mean (s.d.) & Median (range) & Mean (s.d.) & Median (range) & \\
\hline Global QOL & $69(24)$ & $71(25-100)$ & $65(28)$ & $71(0-100)$ & 0.86 \\
\hline \multicolumn{6}{|l|}{ Functional scale } \\
\hline Social function & $75(28)$ & $83(33-100)$ & $80(24)$ & $92(17-100)$ & 0.71 \\
\hline Cognitive function & $67(35)$ & $75(0-100)$ & $82(23)$ & $83(17-100)$ & 0.26 \\
\hline Role function & $69(31)$ & $67(17-100)$ & $83(24)$ & $100(17-100)$ & 0.30 \\
\hline Emotional function & $73(34)$ & $83(0-100)$ & $81(22)$ & $88(33-100)$ & 0.64 \\
\hline Physical function & $78(19)$ & $83(47-100)$ & $81(24)$ & $93(20-100)$ & 0.47 \\
\hline \multicolumn{6}{|l|}{ Symptom scale } \\
\hline Pain & $25(24)$ & $25(0-50)$ & $18(29)$ & $0(0-100)$ & 0.36 \\
\hline Fatigue & $36(37)$ & $33(0-100)$ & $25(23)$ & $22(0-67)$ & 0.57 \\
\hline Nausea and vomiting & $10(15)$ & $0(0-33)$ & $3(6)$ & $0(0-17)$ & 0.30 \\
\hline \multicolumn{6}{|l|}{ Single items } \\
\hline Dyspnoea & $21(40)$ & $0(0-100)$ & $8(24)$ & $0(0-100)$ & 0.64 \\
\hline Insomnia & $21(35)$ & $0(0-100)$ & $33(29)$ & $33(0-100)$ & 0.22 \\
\hline Appetite loss & $13(25)$ & $0(0-67)$ & $3(10)$ & $0(0-33)$ & 0.53 \\
\hline Diarrhoea & $17(36)$ & $0(0-100)$ & $17(25)$ & $0(0-100)$ & 0.67 \\
\hline Constipation & $17(18)$ & $17(0-33)$ & $15(23)$ & $0(0-67)$ & 0.71 \\
\hline Financial impact & $29(33)$ & $17(0-67)$ & $17(25)$ & $0(0-67)$ & 0.41 \\
\hline
\end{tabular}

of life of patients, were similar. There was no difference in medians for all other scale scores. Patients having had APR seem to have less sleep disturbances (0 versus 33; $\mathrm{p}=0.22$ ) and they tended to report lower levels of role functioning (67 versus 100; $\mathrm{p}=0.3$ ) and cognitive functioning (75 versus $83 ; \mathrm{p}=0.26$ ) than did patients having had restorative resection.

The results of QLQ-C38 for the two surgical groups are given in Table 3 . No significant differences in median scores were observed between the two surgical groups for any of the scales. However, APR group of patients tended to report a lower body image score (61 versus $89 ; \mathrm{p}=0.16$ ). The sexual functioning score and sexual enjoyment score were very low in both groups, but in the APR group the sexual functioning score was higher (33 versus $17 ; \mathrm{p}=0.11$ ).

\section{Discussion}

The abdominoperineal resection (APR) was long considered the standard treatment of tumours lying in the lower third of the rectum, providing a good local control. A more precise understanding of tumour biology and of failure patterns, has lead to the acceptance of short distal resection margins. Advances in surgical stapling and coloanal anastomoses technique have made it possible to treat many low rectal cancers by the sphincter-saving low anterior resection in preference to an APR. The survival and lo- 
Table 3. EORTC QLQ-C38 mean and median functional scale and single - item scores according to the type of surgery

\begin{tabular}{lccccc}
\hline & \multicolumn{2}{c}{$\begin{array}{c}\text { APR } \\
\text { (8 patients) }\end{array}$} & $\begin{array}{c}\text { Restorative surgery } \\
\text { (20 patients) }\end{array}$ \\
Item & Mean (s.d.) & Median (range) & Mean (s.d.) & Median (range) & $P$ \\
\hline Functional scale & & & & & \\
$\quad$ Body image & $67(23)$ & $61(33-100)$ & $81(20)$ & $89(44-100)$ & 0.16 \\
$\quad$ Future perspectives & $46(43)$ & $50(0-100)$ & $56(35)$ & $67(0-100)$ & 0.56 \\
$\quad$ Sexual functioning & $40(32)$ & $33(0-100)$ & $19(21)$ & $17(0-67)$ & 0.11 \\
$\quad$ Sexual enjoyment & $44(34)$ & $33(0-100)$ & $29(28)$ & $33(0-67)$ & 0.49 \\
Symptom scale & & & & & \\
Micturition problems & $33(28)$ & $33(0-78)$ & $22(22)$ & $11(0-56)$ & 0.39 \\
General gastrointestinal & $17(21)$ & $7(0-60)$ & $19(14)$ & $20(0-40)$ & 0.48 \\
Defecation problems & & & $23(17)$ & $24(0-57)$ & \\
Stoma- related problems & $26(20)$ & $21(5-67)$ & & & \\
Sexual dysfunction of males & $0(0)$ & $0(0-0)$ & $0(0)$ & $0(0-0)$ & 1.0 \\
Sexual dysfunction of females & 17 & $17(17-17)$ & $17(24)$ & $17(0-33)$ & 1.0 \\
Weight loss & $8(15)$ & $0(0-33)$ & $5(17)$ & $0(0-67)$ & 0.62 \\
\hline
\end{tabular}

cal recurrence rate was not compromised. ${ }^{9}$

There are many other factors, which impact the decision, which surgical procedure to undertake for low-lying cancers: patient gender, preoperative sphincter function, stage of the disease, potential distal resection margin and surgeon preference. The avoidance of permanent colostomy has been used to judge the quality of the rectal cancer surgery. Although the avoidance of a permanent stoma following rectal cancer excision is regarded as a favourable outcome measure, the bowel function after the sphincter-sparing procedures may be greatly altered, resulting in faecal urgency and incontinence. ${ }^{10}$ Patients receiving preoperative radiochemotherapy for rectal cancer may develop also other unpleasant symptoms, such as micturition problems and sexual dysfunction. These symptoms, which occurred in a substantial proportion of patients, have been reported previously. ${ }^{11}$ Our aim was to evaluate the effect of these symptoms on the health-related QOL as an important endpoint.

It is difficult to evaluate the QOL after the rectal cancer surgery. For that purpose, non-cancer-specific or nonstardized questionnaires with a different methodology for scoring were used, i.e. self-reported by patients or scored by physicians. Additionally, the authors provided different types of preoperative or postoperative treatment. The evaluations of QOL were mostly of retrospective nature with a different time for questionnaire administration and evaluated on small sample sizes. So, the heterogeneity in the evaluation of QOL after the rectal cancer surgery gave rise to inconsistent and conflicting findings. Any comparison between data reported by different authors might be misleading. The use of standardized questionnaires is necessary. In our study, the evaluation of health-related QOL of patients was assessed by using EORTC cancer and site specific questionnaires, 
which are validated and preferred measures in recent clinical trials.

Some studies have suggested that patients with a colostomy have a poorer QOL when compared to those who had restorative resection. ${ }^{12,13}$ In the present study no significant differences in median scores were observed in any of the function scales of QLQ-C30 questionnaire studied (physical, role, social, emotional, cognitive functions and overall QOL) between the two groups. Our finding is in agreement with the observation reported by Allal et al. ${ }^{14}$ and Camilleri-Brennan et al. ${ }^{4}$ In a prospective study, Grumann et al. ${ }^{15}$ showed that following LAR patients had even a lower QOL than those who underwent APR. A recent analysis of eight studies in a Cochrane Database Systemic Review showed mixed results. Half of the studies revealed no difference with regard to QOL between APR and LAR, in one the QOL in patients with stoma was only slightly affected and others revealed that the formation of stoma significantly affected the patients QOL. ${ }^{16}$ The similarities in QOL in stoma and nonstoma patients may be due to the adaptation and the phenomenon of "response shift", ${ }^{\prime \prime}$ in which patients who have survived life-threatening disease seem to have new internal standards and, thereby, often report good QOL. Even patients who have undergone pelvic exenteration report having good QOL. ${ }^{18}$ In agreement with other authors, we found that APR was associated with a lower perception of body image (feeling less attractive) than LAR.

Defecation-related problems, such as urgency, incontinence and incomplete bowel emptying, are well-known side effects of sphincter-preserving surgery. ${ }^{11}$ Interestingly, the assessment of gastrointestinal problems on the QLQ-C38 and constipation on the QLQ-C30 showed similarities in the two groups. Camilleri-Brennan et al. found that patients who had sphincter saving resection had more problems with constipation. $^{4}$ Scores for stoma-related problems in both studies were comparable low, probably due to better stoma care. Standardized training by the specialized nurse is performed in every stoma patient in our department. That might lead to a better perception of QOL in our study. ${ }^{19}$

While most of the studies have suggested that the sexual function was impaired in patients receiving permanent stomas, $15,20,21$ in present study, no difference in this dimension of the QLQ-C38 was revealed. Unexpected, the sexual functioning score tended to be higher in patients with APR than in patients with LAR, although the difference was not significant. Because of a small sample size and older age of our patients than in other studies, no valid conclusion can be made regarding this issue. Urinary problems were more frequently encountered after APR than after LAR. These differences are probably surgeon dependent.

The present study is limited by a lack of control measurements before the treatment. In addition, the number of evaluated patients was small, as only patients without evidence of disease treated in one clinical trial were surveyed. So a lack of statistical power might also be relevant. To obtain a large, unselected patient sample, we started to evaluate the QOL of all patients with rectal cancer, treated with preoperative radiochemotherapy, prospectively: before preoperative treatment, 1 year and 3 years after the operation.

In conclusion, consequences of the multimodality treatment of rectal cancer have an important bearing on QOL. Patients after the combined modality treatment with restorative surgical procedures do not necessary have a better QOL, mainly due to the impairment of the bowel function. In addition to traditional endpoints, such a disease control and survival, assessing restrictions 
in QOL are necessary to provide a comprehensive understanding of the outcome of the combined modality treatment.

\section{References}

1. Sauer R, Becker H, Hohenberger W, Rodel C, Wittekind C, Fietkau R, et al. Preoperative versus postoperative chemoradiotherapy for rectal cancer. N Engl J Med 2004; 351: 1731-40.

2. Dahlberg M, Glimelius B, Graf W, Phlman L. Preoperative irradiation affects functional results after surgery for rectal cancer: Results from a randomised study. Dis Colon Rectum 1998; 41: 54349.

3. Sofić A, Šehović N, Bešlić Š, Prnjavorac B, Bilalović $\mathrm{N}$, Čaluk J, et al. MR rectum imaging with ultra sound gel as instrumental contrast media in tubulovillous adenoma. Radiol Oncol 2008; 42: 136-42.

4. Camilleri-Brennan J, Cteele RJC. Objective assessment of morbidity and quality of life after surgery for low rectal cancer. Colorectal Dis 2002; 4: 61-6.

5. Velenik V, Anderluh F, Oblak I, Strojan P, Zakotnik $B$. Capecitabine as a radiosensitizing agent in neoadjuvant treatment of locally advanced resectable rectal cancer: prospective phase II trial. Croat Med J 2006; 47: 693-700.

6. Aaronson NK, Ahmedzai S, Bergman S, Bullinger M, Cull A, Duez NJ, et al. The European Organisation for Research and Treatment of Cancer QLQ-C30; A quality of life instrument for the use in international clinical trials in oncology: J Natl Cancer Inst 1993; 85: 365-76.

7. Sprangers MAG, te Velde A, Aaronson NK. The construction and testing of the EORTC Colorectal Cancer Specific Quality-of-life questionnaire Module (the QLQ-C38). Eur J Cancer 1999; 35: 23847.

8. Fayers P, Aaronson N, Bjordal K, Curran D, Groenvold M. EORTC QLQ-C30 Scoring Manual. $2^{\text {nd }}$ edition. Brussels: EORTC Quality of Life Study Group; 1999.

9. Williams NS, Johnston D. Survival and recurrence after sphincter saving resection and abdominoperineal resection for carcinoma of the middle third of the rectum. Br J Surg 1984; 71: 278-82.

10. Di Betta E, D'Hoore A, Filez L, Penninckx F. Sphincter saving rectum resection is the standard procedure for low rectal cancer. Int J Colorectal Dis 2003; 18: 463-9.
11. Velenik V, Anderluh F, Oblak I, Strojan P, Segedin B, Zakotnik B. Preoperative capecitabine and concomitant radiotherapy in operable rectal cancer: a phase II study with 2 years follow-up. Science and multidisciplinary management of GI malignancies: Proceedings book 2008. p. 325. [Abstract No 516].

12. Williams NS, Johnston D. The quality of life after rectal excision for low rectal cancer. Br J Surg 1983; 70: 460-2.

13. Frigell A, Ottander M, Stenbeck H, Pahlman L. Quality of life of patiemts treated with abdominoperineal resection or anterior resection for rectal carcinoma. Ann Chir Gynaecol 1990; 79: 26-30.

14. Allal AS, Bieri S, Pelloni A, Spataro V, Anchisi S, Ambrosetti $\mathrm{P}$, et al. Sphincter-sparing surgery after preoperative radiotherapy for low rectal cancers: feasibility, oncologic results and Quality of life outcomes. Br J Cancer 2000; 82: 1131-7.

15. Grumann MM, Noack EM, Hoffmann IA, Schlag PM. Comparison of quality of life in patients undergoing abdominoperineal extirpation or anterior resection for rectal cancer. Ann Surg 2001; 233: 149-56.

16. Pachler J, Wille-Jorgensson P. Quality of life after rectal resection for cancer, with or without permanent colostomy. Cochrane Database Syst Revue 2005; 18: CD004323.

17. Sprangers MA, Schwartz CE. The challenge of response shift for quality-of-life-based clinical oncological research. Ann Oncol 1999; 10: 747-9.

18. Guren MG, Wiig JN, Dueland S, Tveit KM, Fossa SD, Waehre $\mathrm{H}$, et al. Quality of life in petients with urinary diversion after operation for locally advanced rectal cancer. Eur J Surg Oncol 2001; 27: 645-51.

19. Comb J. Role of stoma care nurse: patients with cancer and colostomy. Br J Nurs 2003; 12: 852-6.

20. Sprangers MAG, Taal BG, Aaronson NK, te Velde A. Quality of life in colorectal cancer. Stoma vs. nonstoma patients. Dis Colon Rectum 1995; 38: 3619.

21. Schmidt CE, Bestmann B, Kuchler T, Longo WE, Kremer B. Prospective evaluation of quality of life of patients receiving either abdominoperineal resection or sphincter-preserving procedure for rectal cancer. Ann Surg Oncol 2005; 12: 117-23. 